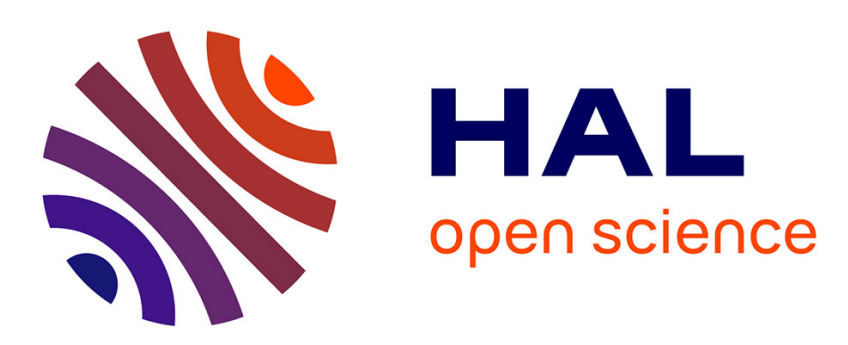

\title{
Characterisation of coherent rotating modes in a magnetised plasma column using a mono-sensor tomography diagnostic
}

Pierre David, Alexandre Escarguel, Yann Camenen, Romain Baude

\section{To cite this version:}

Pierre David, Alexandre Escarguel, Yann Camenen, Romain Baude. Characterisation of coherent rotating modes in a magnetised plasma column using a mono-sensor tomography diagnostic. 2016. hal-01344867

\section{HAL Id: hal-01344867 \\ https://hal.science/hal-01344867}

Preprint submitted on 12 Jul 2016

HAL is a multi-disciplinary open access archive for the deposit and dissemination of scientific research documents, whether they are published or not. The documents may come from teaching and research institutions in France or abroad, or from public or private research centers.
L'archive ouverte pluridisciplinaire HAL, est destinée au dépôt et à la diffusion de documents scientifiques de niveau recherche, publiés ou non, émanant des établissements d'enseignement et de recherche français ou étrangers, des laboratoires publics ou privés. 


\title{
Characterisation of coherent rotating modes in a magnetised plasma column using a mono-sensor tomography diagnostic
}

\author{
P. David, ${ }^{1, a)}$ A. Escarguel, ${ }^{1, b)}$ Y. Camenen, ${ }^{1}$ and R. Baude ${ }^{1}$ \\ Aix-Marseille Université, St Jérôme, CNRS, PIIM UMR7345, case 322, 13397 Marseille, \\ France
}

(Dated: 11 July 2016)

In this paper we report on an original tomographic diagnostic using a single sensor on a magnetized plasma column. The experimental set-up and the numerical inversion method used to interpret the data are presented. The core plasma evolution during the rotation of coherent modes is characterized with this tomography diagnostic. The experimental observations show that the mode shape is constant during the plasma rotation. The results are also compared to two-dimensional probe measurements.

PACS numbers: 42.30.Wb, 52.35.Py, 52.70.Kz, 52.75.Xx

\section{INTRODUCTION}

Over the past few years, several linear devices have been investigating the physics of coherent rotating structures, helped with recent developments in both technology and theory. Plasmas with cross field configurations are present in many domains, including Hall effect thrusters, ion sources and magnetic fusion. These configurations can lead to various types of nonlinear instabilities among which coherent rotating structures have been observed frequently ${ }^{1-5}$. In this paper, we present a new way of monitoring the magnetized plasma of the Mistral device ${ }^{6}$ sketched in figure 1. An Argon plasma created by a thermoionic discharge, with a typical pressure $P \simeq 10^{-2} \mathrm{~Pa}$ and electron density $n_{\mathrm{e}} \simeq 10^{15} \mathrm{~m}^{-3}$, and temperature $T_{\mathrm{e}} \simeq 4 \mathrm{eV}$ is studied. The device is separated in two zones: the source chamber, and the linear plasma chamber. The source chamber consists of 32 heated tungsten filaments emitting electrons with an energy of a few tens of electron-volts (primary electrons), with randomized trajectories through a magnetic cusp configuration. The primary electrons are injected in the second chamber through an $80 \mathrm{~mm}$ diameter diaphragm. As a consequence, the central plasma column is slightly electronegative. The linear chamber, where we study the plasma, is a $1.2 \mathrm{~m}$ long and $0.4 \mathrm{~m}$ wide cylinder with a $16 \mathrm{mT}$ axial magnetic field. The plasma is limited by a grid at each end of the column (called collector and separating grid in the figure 1) and is surrounded by a $20 \mathrm{~cm}$ wide cylinder, each of them can be polarized to control electrons confinement.

Global modes with azimuthal numbers $m=1$ or $m=2$ are routinely observed on Mistral ${ }^{7-11}$ and can be kept steady for several hours. These modes have been interpreted $^{12}$ as Simon-Hoh ${ }^{13,14}$ instabilities with null axial numbers $\left(k_{\|}=0\right)$. A simplified fluid model based on the azimuthal momentum equation in the presence of a

\footnotetext{
a) pierre.david@univ-amu.fr

b) alexandre.escarguel@univ-amu.fr
}

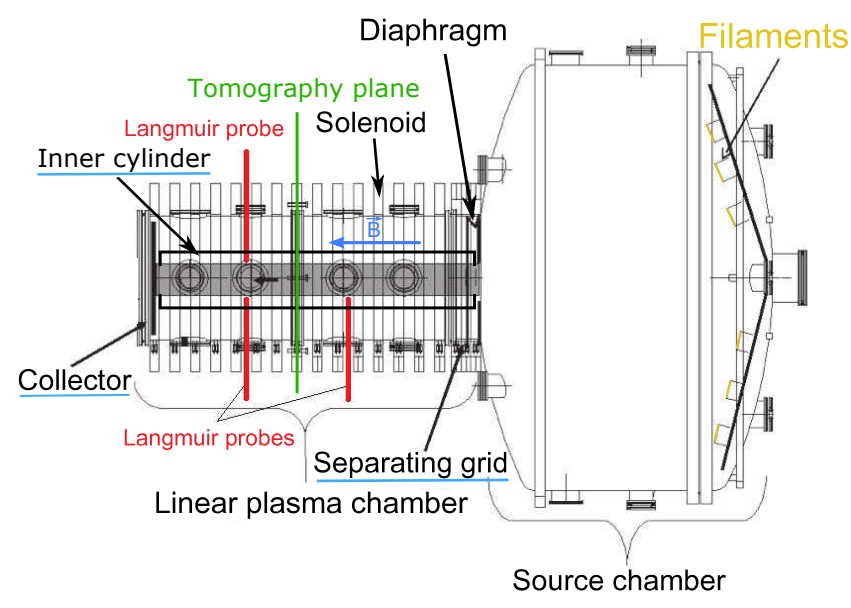

FIG. 1. The Mistral linear plasma device. The blue underlined elements can be individually polarized, controlling the longitudinal (collector and separating grid) and the radial (inner cylinder) boundary conditions of the plasma.

radial electric field qualitatively reproduces the dependences of the mode rotation frequency on the pressure and the magnetic field ${ }^{15}$. Primary electrons play an important role in the apparition of those highly non-linear modes by creating a radial electric field between the negative plasma and the wall ${ }^{8,9}$. Consequently, the radial electric field is expected to be able to expel energetic electrons in the limiter's shadow that will ionise a plasma arm. Therefore the boundary conditions play a key role in the instability existence by balancing the axial and radial confinement in order to incite the electrons to be radially ejected from the column.

Most experimental works on linear devices have been performed with electrostatic probes assuming that the mode structure is constant over a period of rotation. Despite the fact that tomography diagnostics are seldom used in low temperature plasma experimental studies, due to price and dimensions, this technique can bring new insight on plasma physics. Tomographic analysis is non-invasive, temporally resolved acquisition is easily 


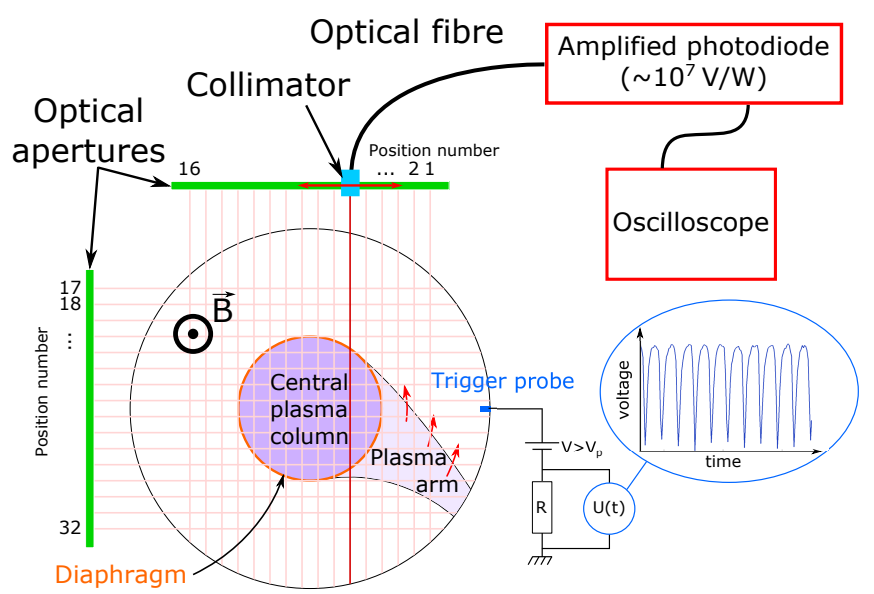

FIG. 2. Single sensor tomography set-up (mid-plane section of the column).

achievable and no hypothesis is required regarding the plasma shape and motion. The experimental set-up and the associated numerical tomographic inversion are presented in section II, before we analyse the results in section III. They are finally compared to $2 \mathrm{D}$ reconstructions of the plasma using a Langmuir probe in section IV.

\section{MONO-SENSOR TOMOGRAPHY SET-UP}

Taking advantage of the rotating mode long life span (up to 2-3 hours - limitation imposed by the source chamber increasing temperature), tomography is performed using conditional sampling with a single sensor moved to different positions (figure 2). The acquisition at each position is synchronized with the plasma rotation through the trigger probe (polarised at $V>V_{\mathrm{p}}$ ) signal. The trigger occurs when an excess of current is detected, meaning that the probe is seeing the plasma rotating arm at a specific position. The sensor is an amplified photodiode with a $10^{7} \mathrm{~V} / \mathrm{W}$ gain, giving a sufficient sensitivity and a good signal/noise ratio. We separate the sensor from the plasma using an optical fibre in order to prevent any magnetic perturbation and to have a higher mobility of the optomechanical system.

The acquisition of signals for tomography with a single sensor requires its displacement to different calibrated positions. In order to limit associated errors, we only used a single movement, giving three possible chords setups. The first is a translation of the collimated optical fibre leading to parallel chords. The second is a translation of the bare fibre behind a pinhole, giving fan distributed chords. Finally, the third option is to reproduce the same fan distribution with a rotation of the collimated fibre. The fan distribution is known to lessen the effect of noise by providing more viewing angles ${ }^{16}$. However, the pinhole reduces the available luminosity by a factor 25 , greatly decreasing the signal/noise ratio, and looking at the whole plasma with the rotative system

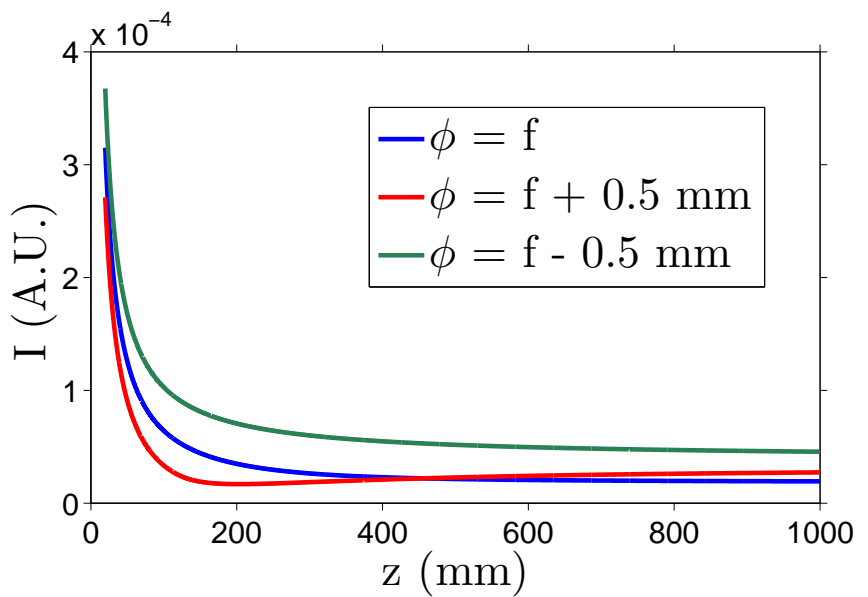

FIG. 3. Variation of the coefficient $I(z)$ weighting the transfer matrix, for different positions of the sensor (here the optical fibre) $\phi$ regarding the lens' focal length $f$. Here $f=10.9 \mathrm{~mm}$.

would require the fibre holder to be placed through the vacuum chamber window. The parallel configuration has therefore been preferred. The plasma facing end of the fibre is collimated with a lens to a conical line of sight from 8 (at the lens) to $15 \mathrm{~mm}$ (at the opposite wall) wide. The number of positions (tomography's lines of sight) is chosen so that the viewing cones do not intersect their neighbours before the wall, yielding $2 \times 16$ lines of sight through the optical apertures. The plasma's emissivity is recorded at each position during about $2 \mathrm{~ms}$ (about 10 mode rotations). For each position, the measurement is repeated 16 times and then averaged to increase statistics.

The numerical tomographic inversion is based on a finite-element scheme. The cross-section of interest is divided into $N$ pixels whose emissivity $E=\left(e_{j}\right)_{j \in[1 . . \mathrm{N}]}$ contributes to the 32 integrated measured signals $S=$ $\left(s_{i}\right)_{i \in[1 . .32]}$. $E$ and $S$ are related by the transfer matrix $T=\left(t_{i j}\right)$ depending on the geometry of the system and the response of the detector. By inverting the system $T . E=S$, the local emissivity can be retrieved from the line integrated measurements. The transfer matrix is determined by calculating and discretising the power $P$ received by the sensor from each plasma element $\mathrm{d}^{3} \mathbf{r}$ :

$$
P=\iiint_{\mathrm{V}_{\text {chord }}} \int_{\Lambda} e(\mathbf{r}, h \nu) \mathcal{E}(h \nu) \frac{\Omega_{\text {lens }}(\mathbf{r})}{4 \pi} \mathrm{d}(h \nu) \mathrm{d}^{3} \mathbf{r}
$$

in which $\mathrm{V}_{\text {chord }}$ is the volume of the chord, supposed here to be conical, $\Lambda$ is the sensor spectral range, and $\Omega_{\text {lens }}(\mathbf{r})$ is the solid angle of the lens seen from the plasma element $\mathrm{d}^{3} \mathbf{r}$. In the integrand, $e$ and $\mathcal{E}$ are the emissivity of the element $\mathrm{d}^{3} \mathbf{r}$ and the sensor spectral response respectively, with the three dimensional position vector $\mathbf{r}$ and the energy variable $h \nu$. For an optically thin plasma and lines of sight defined by a finite sensor behind a simple aperture $^{17,18}$, each element $t_{i j}$ is given by the length $l_{i j}$ 
of the $i^{\text {th }}$ chord in the $j^{\text {th }}$ pixel multiplied by a calibration constant $A$ : $T=A$. $\left(l_{i j}\right)$. However, when a lens is used for the collimation, some simplifications cannot be made, and the transfer matrix elements become

$$
t_{i j}=l_{i j} . I_{i j}
$$

where $I$ is a function of the distance $z$ between the lens and the pixel, depending on the lens size and focal length, the sensor (here the optical fibre) size, and the distance between the sensor and the lens. Figure 3 shows the variation of $I(z)$ for several distances $\phi$ between the lens and the sensor, in the experimental configuration used here. With variation of only $0.5 \mathrm{~mm}(\leqslant 5 \%$ of the focal length), there is an important variation of $I(z)$, which is also different for either side of the focal point. In order to ensure which function had to be used for the transfer matrix, the optical fibre was placed at $\phi=f-\varepsilon_{\mathrm{f}}$, with $\varepsilon_{\mathrm{f}}=0.3 \mathrm{~mm}$. This way potential errors on the placement would not change the slope of $I(z)$ for $z$ in our range of interest (200 to $400 \mathrm{~mm}$ ), so without an absolute calibration the result would remain unchanged.

To solve this large, ill-defined and underdetermined system, the Tikhonov regularisation is used, so solutions minimising

$$
\frac{1}{2}(T \cdot E-S)^{2}+\alpha \mathcal{R}
$$

with $\alpha$ a positive weighting parameter and $\mathcal{R}$ a regularising functional, are sought for. Several inversion methods were tested on arbitrary signals and on ghost images, having in mind future analyses on non-coherent modes using an upgraded tomography set-up being installed on Mistral. Among zero, first and second order, minimum Fisher information and SVD regularisations ${ }^{19}$, the second order spatial derivative proved to have the most consistent results and a low sensitivity to noise in our case. Figure 4 shows a ghost image (left), its discretisation (top-right) and the tomography result associated (bottom-right). The average error between each of their pixels is between $10 \%$ and $15 \%$ for different ghost images with up to $15 \%$ random noise.

In order to perform the tomographic inversion, the following hypotheses are made:

(H1) The chords are cone-shaped, defined in cylindrical coordinates $(r, \theta, z)$ by

$$
\left\{r \in\left[0, r_{\max }(z)\right], \theta \in[0,2 \pi], z \in\left[0, z_{\max }\right]\right\}
$$

with $z$ the distance to the lens, $z_{\max }$ the distance between the lens and the wall, and $r_{\max }(z)$ the radius of the line of sight at position $z$.

(H2) In any given chord, the emissivity is constant in a $(r, \theta)$ disk: $e(\mathbf{r}, h \nu) \simeq e(z, h \nu)$.

(H3) The solid angle of the lens seen from any point of those disks is constant: $\Omega_{\text {lens }}(\mathbf{r}) \simeq \Omega_{\text {lens }}(z)$.

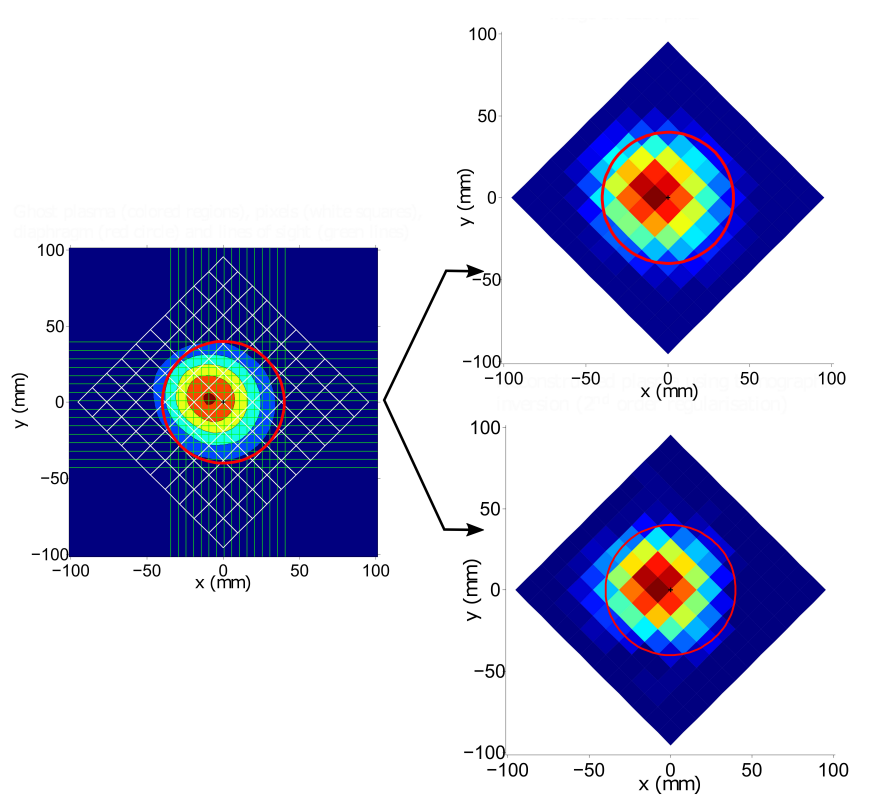

FIG. 4. Ghost plasma image (left) and its discretisation (top right) compared to its tomographic reconstruction (bottom right). On the ghost image the pixels used in tomography are shown in white, the lines of sight for the artificial measurements arein green, and the red circle represents the diaphragm.

(H4) The emissivity is constant inside a pixel.

(H5) The emissivity is zero where there is no pixel.

(H6) Pixels are only linked by the minimisation of the second order spatial derivative, present in $\mathcal{R}$.

Some comments on those hypotheses are in order. (H1) only assumes no irregularity in the circular symmetry of the optical system. (H2) supposes that the chords' spread is small enough compared to the pixels size. If the characteristic sizes of the lens (radius and focal length) and its line of sight's radius are much smaller than the distance at which the plasma is seen, (H3) is respected. (H4) requires to have small enough pixels, but the number of pixels (and consequently their size) is limited by the number of measurements plus the regularisation method. Finally, the combination of (H2), (H3) and (H4) points out the need for an accurately collimated system.

For the experimental conditions considered in this paper, the plasma spectrum is largely dominated by neutral argon emission lines ${ }^{9}$. With the low plasma densities in Mistral $\left(<10^{15} \mathrm{~m}^{3}\right)$, the coronal model can be used ${ }^{20}$. In these conditions, the intensity of the neutral emission lines is proportional to the thermal electrons' density $n_{\mathrm{e}, \mathrm{th}}$ and the primary electrons' density $n_{\mathrm{e}, \mathrm{p}}$, so the calculated local emissivity $E$ is a linear combination of $n_{\mathrm{e}, \mathrm{th}}$ and $n_{\mathrm{e}, \mathrm{p}}: E=a \cdot n_{\mathrm{e}, \mathrm{th}}+b \cdot n_{\mathrm{e}, \mathrm{p}}$, with $a$ and $b$ functions of the energy of the thermal and primary electron respectively and the excitation cross sections of Argon by collision with electrons. 


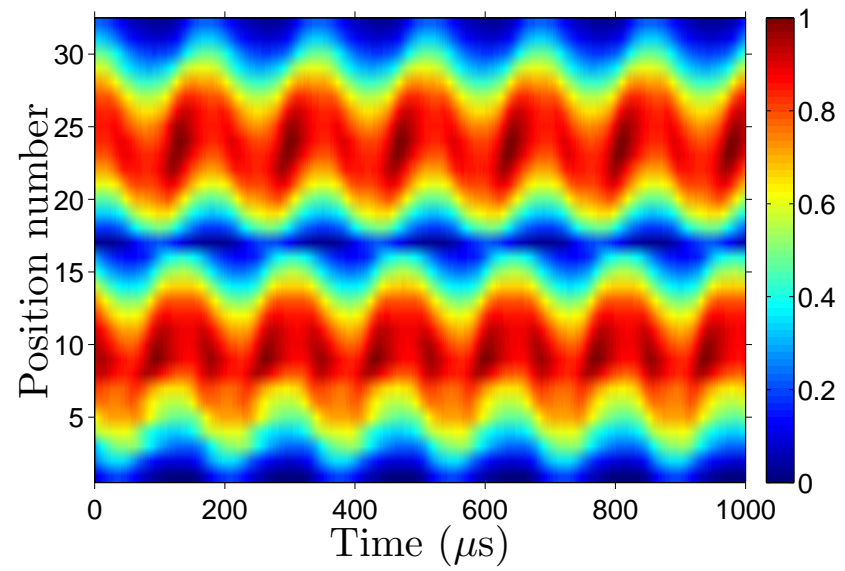

FIG. 5. Raw time evolution (horizontal axis) of the signal from each position (vertical axis) during $1 \mathrm{~ms}$. See figure 2 for the position numbering. The signal is normalized to the highest value of the whole acquisition.

\section{EXPERIMENTAL TOMOGRAPHY RESULTS}

In this section, we will use the system described previously to study the behaviour of the coherent mode during its rotation.

The time evolution of the raw measurements at every positions is shown in figure 5 , with the positions number 1 to 16 from the top of the plasma and 17 to 32 from the side, as displayed in figure 2 . The sinusoidal oscillation of the mode is clearly seen, with a phase shift of $+\pi / 4$ between the top and the lateral measurements indicating a anticlockwise rotation (looking at the plasma from the left side on figure 1). The mode frequency is $f \simeq 5.8 \mathrm{kHz}$. An intensity asymmetry between the rising edge and the falling edge of the oscillation, i.e. when the sensor sees the plasma arm is in front of and behind the central plasma respectively, is noticeable, especially for the lateral aperture (positions number 17 to 32 ). This asymmetry is due to the geometrical factor $I$ discussed in the previous section. The plasma being between 200 and $400 \mathrm{~mm}$ from the lens, the decrease in $I$ exactly compensates for the asymmetry of the signal and the different asymmetries between the top and lateral positions come from the top measurements being made a couple centimetres further from the plasma, where $I(z)$ is flatter.

Tomographic inversion is applied at each time step to reconstruct the evolution of the local plasma emissivity. To increase readability, we separate the emissivity into the temporal average and fluctuations component:

$$
E=\langle E\rangle_{t}+\delta E,
$$

and show in 6-(a) $\langle E\rangle_{t}$ normalised to the value at the plasma center, and in 6 -(b) the emissivity variations relatively to the time average. The frames are separated by $45.6 \mu \mathrm{s}$, for a total of about $80 \%$ of a whole period. The time evolution of the emissivity profile visually confirms that the mode remains constant during its rotation.

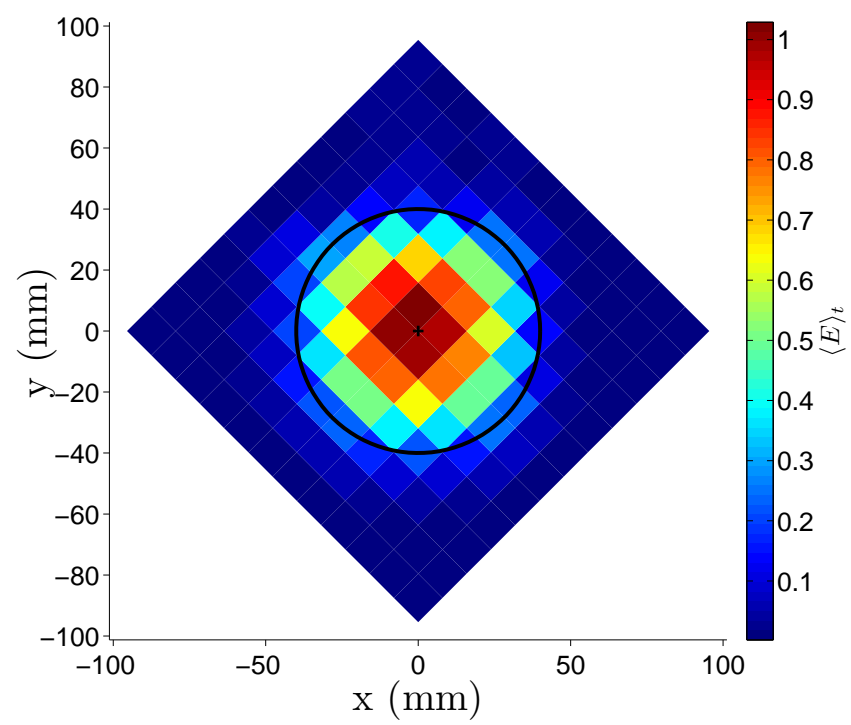

(a)

(b)
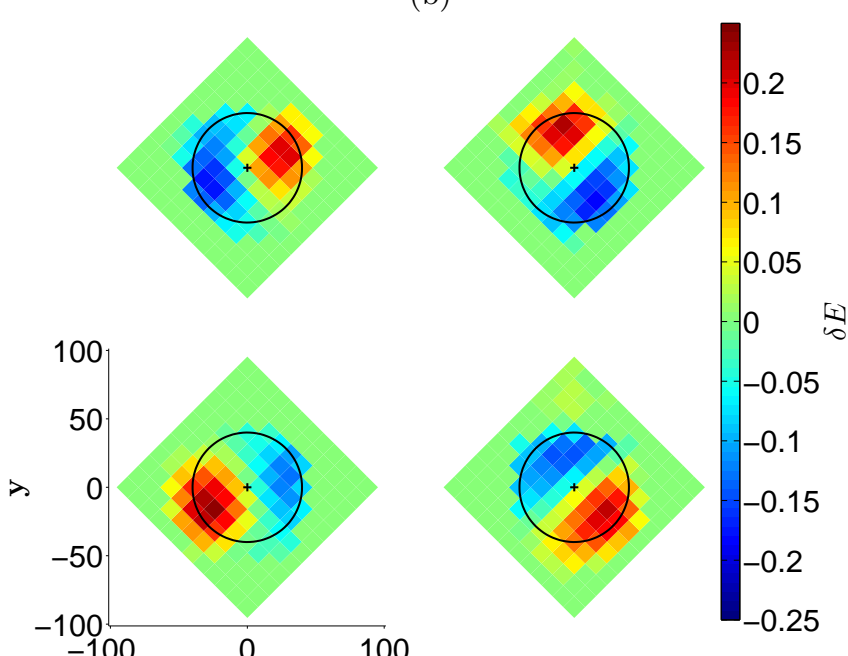

FIG. 6. Tomographic inversion results: (a) temporal average emissivity over the whole acquisition normalized to the central point of the plasma. (b) Emissivity variation at four different times of a single period. The black circle represents the limiter. The spatial scale is in millimetres.

In order to compile the spatial evolution during the whole acquisition into a single figure, the azimuthal emissivity profiles are extracted at several radii and phase shifted to have their maxima aligned for all time steps, and the average over all time steps is calculated and displayed in figure 7 . The information about the time evolution of the coherent mode is contained in the error bars, representing the standard deviation. The figure 7 shows the small standard deviation of the azimuthal shape of the mode, confirming that the mode remains constant during its rotation. Additionally, the same dis- 


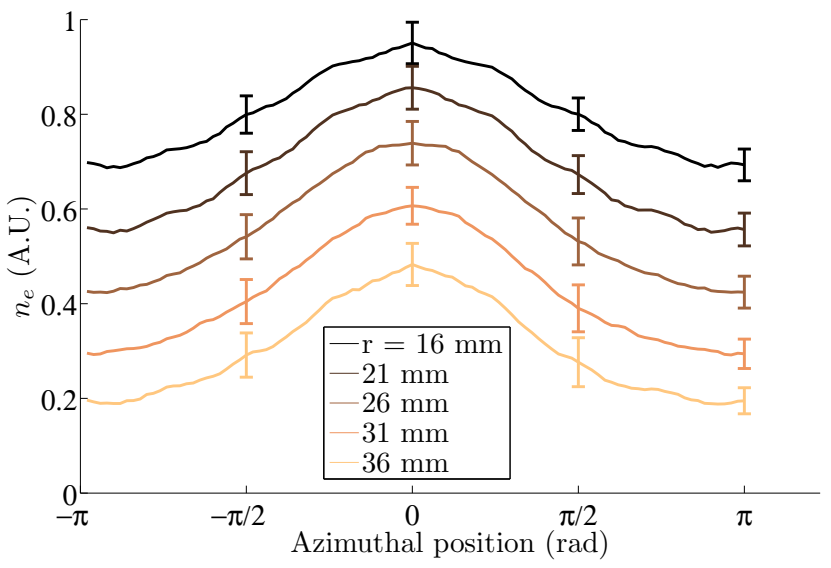

FIG. 7. Averaged emissivity over the whole rotation of the tomographic reconstruction of the experimental measurements as a function of the azimuthal angle at different radii. The errorbars represent the standard deviation during the whole rotation of the plasma.

play method can be applied to ghost images whose time evolution is simulated by a numerical rotation, in which case the same error bars appear, indicating that those variations mainly come from numerical noise.

\section{COMPARISON BETWEEN TOMOGRAPHY AND LANGMUIR PROBE MEASUREMENTS}

In addition to tomography, a radially movable cylindrical Langmuir probe has been used to characterise the coherent rotating structures. The probe acquisitions were triggered using a second probe polarized above the plasma potential, as in the previous section. There are two main downsides to this diagnostic: it is intrusive, and a radially movable probe can a priori only give information on the time evolution at different radii. Yet, reconstructing the azimuthal density profile using a delayed trigger is possible under the hypothesis of the plasma uniformity during its rotation, which was validated by the tomography results of the figure 6 . Two-dimensional probe measurements (azimutal and radial) were therefore performed. The electron density and temperature, and the plasma potential, respectively $n_{\mathrm{e}}, T_{\mathrm{e}}$ and $V_{\mathrm{p}}$, were computed from the probe measurements. However, the magnetic field and the primary electrons lead to a highly anisotropic and bi-Maxwellian plasma. Therefore, we used the Druyvestein formula ${ }^{21}$ to reconstruct the electron energy distribution function $f$ from the second derivative of the $I(V)$ curve (current versus polarization). Densities and temperatures then come from the first and second moments of $f$ respectively. This method has the advantages of being independent of the probe shape (as long as it is convex) and being weakly sensitive to the details of the electron velocities distribution ${ }^{22}$.

The reconstructed section of the electron density and

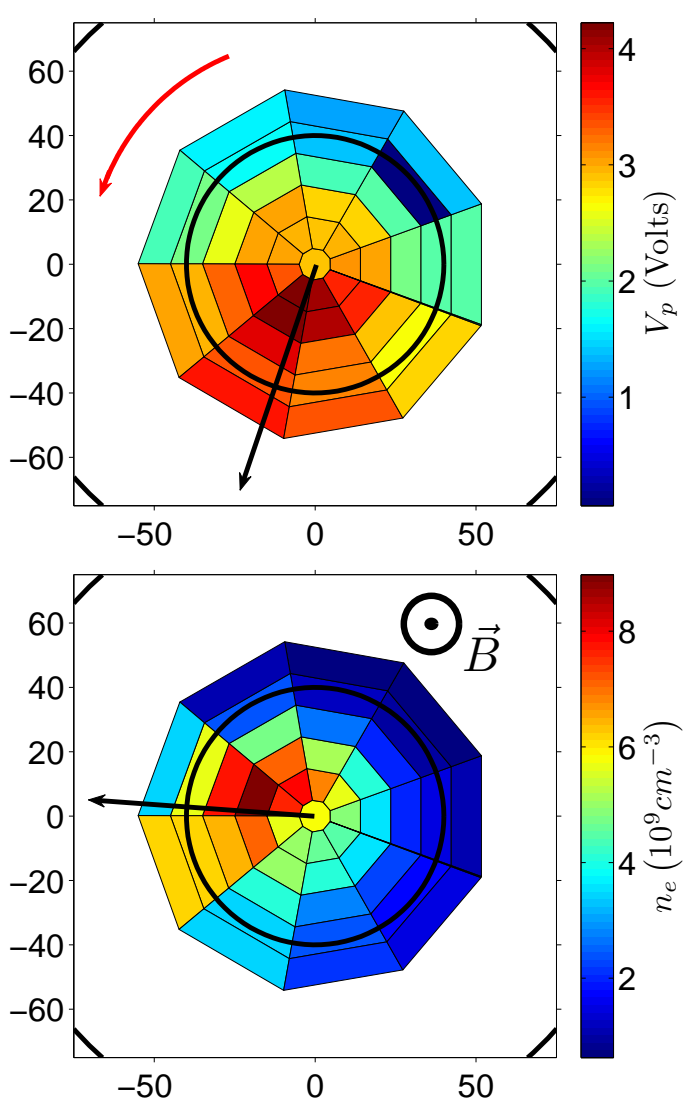

FIG. 8. Two-dimentional probe measurements of the plasma potential $V_{\mathrm{p}}$ and electron density $n_{\mathrm{e}}$. Each area contains the value measured at its center. The red arrow shows the rotation direction of the plasma (counter clockwise). The black circle represents the limiter and the outer circle (visible at each corner) is the polarised wall.

plasma potential is shown in figure 8 . The time delay between two azimuthal positions is $30 \mu$ s and the plasma rotation period is $270 \mu \mathrm{s}$, hence 9 azimuthal measurements for each of the 5 radial positions. The plasma potential and density measurements also display both radial and azimuthal variations in the central column. The plasma potential shifts toward the direction in front of the arm, creating a local electric field inside the limiter in addition to the one at its boundary.

Figure 9 shows the radial variation of the density azimuthal average $\left\langle n_{e}\right\rangle_{\theta}$ and its associated variations $\delta n_{e}$ for both tomography and probe measurements, knowing that under the uniformity hypothesis we have $\left\langle n_{e}\right\rangle_{\theta} \simeq$ $\left\langle n_{e}\right\rangle_{t}$. The density variation $\delta n_{e}(r)$ is taken along the axis passing through the maximum density. Both diagnostics reveal a peaked plasma at the center and a positive and negative shift on each side of the center. However, the tomographic measurements display a narrower averaged density and a lower, but more symmetrical, radial shift than the probe equivalent. Such a difference may be explained by the emissivity being much more sensitive to 


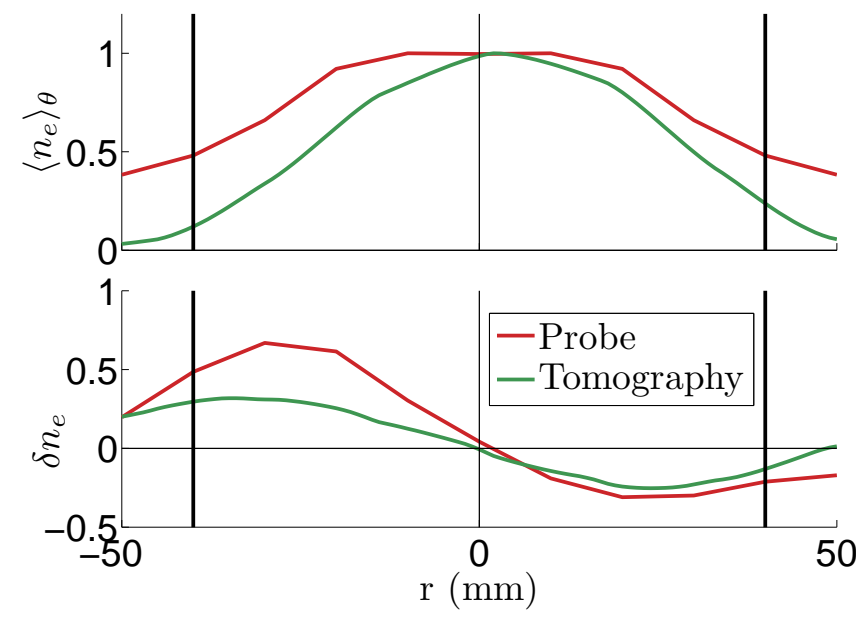

FIG. 9. Radial evolution of the averaged density (top) and density perturbation through the density maximum (bottom) from probe measurements (red) and tomography (green). Values are normalized to the maximum averaged density. Vertical lines show the central region of the plasma.

$n_{e, p}$ than the total density (measured by the electrostatic probe), since $n_{e, t h} \gg n_{e, p}$. Also, primary electrons have a much higher velocity in the parallel direction, so the characteristic times of their perpendicular transport is less important than the parallel transport one, especially compared to the thermal electrons. In order to quantify the contribution of each electron population to the emissivity, the radiative theory would need to be detailed, or the tomography diagnostic would need to be coupled to a spectrometer.

\section{CONCLUSION}

In this work, we show that mono-sensor tomography gives the possibility to reconstruct the local emissivity of the plasma with a simple (regarding both configuration and calibration), low cost and low space demanding diagnostic, especially compared to a full scale tomographic instrument. Contrary to probe diagnostics, it has the advantage to be non intrusive. However, its use is limited to the study of periodic or reproducible events.

It was here used to characterise the time evolution of the emissivity inside the core plasma during coherent rotating modes, demonstrating its steady rotation. A single Langmuir probe could then be used to reconstruct a circular section of the plasma, giving more information on its density and temperature.

Preliminary intensified pictures taken outside of the rotating regime show potential evidence of small scale turbulence which could be at the origin of the instability when the external parameters (mainly boundary conditions and pressure) meet some specific requirements. A full scale tomographic diagnostic is under implementation to study the growth of the rotating modes.

\section{ACKNOWLEDGMENTS}

The authors would like to thank to L. Couëdel for several practical ideas and useful discussions.

This work has been carried out thanks to the support of the $A *$ MIDEX project ( $n^{\circ}$ ANR-11-IDEX-0001-02) funded by the "Investissements d'Avenir" French Government program, managed by the French National Research Agency (ANR).

${ }^{1}$ A. Fredriksen, C. Riccardi, L. Cartegni, and H. Pécseli, Plasma Physics and Controlled Fusion 45, 721 (2003).

${ }^{2}$ G. Y. Antar, J. H. Yu, and G. Tynan, Physics of Plasmas 14, 022301 (2007), http://dx.doi.org/10.1063/1.2424886.

${ }^{3}$ P. Manz, M. Xu, S. H. Müller, N. Fedorczak, S. C. Thakur, J. H. Yu, and G. R. Tynan, Physical Revue Letters 107, 195004 (2011).

${ }^{4}$ T. Kobayashi, S. Inagaki, H. Arakawa, S. Oldenbürger, M. Sasaki, Y. Nagashima, T. Yamada, S. Sugita, M. Yagi, N. Kasuya, A. Fujisawa, S.-I. Itoh, and K. Itoh, Plasma and Fusion Research 6, 2401082 (2011).

${ }^{5}$ O. D. Cortázar, A. Megía-Macías, O. Tarvainen, and H. Koivisto, Physics of Plasmas 22, 123511 (2015), http://dx.doi.org/10.1063/1.4938033.

${ }^{6}$ M. Matsukuma, T. Pierre, A. Escarguel, D. Guyomarc'h, G. Leclert, F. Brochard, E. Gravier, and Y. Kawai, Physics Letters A 314, 163 (2003).

${ }^{7}$ T. Pierre, A. Escarguel, D. Guyomarc'h, R. Barni, and C. Riccardi, Physical Revue Letters 92, 065004 (2004).

${ }^{8}$ S. Jaeger, T. Pierre, and C. Rebont, Physics of Plasmas 16, 022304 (2009), http://dx.doi.org/10.1063/1.3076931.

${ }^{9}$ A. Escarguel, European Physical Journal D 56, 209 (2010).

${ }^{10}$ C. Rebont, N. Claire, T. Pierre, and F. Doveil, Physical Revue Letters 106, 225006 (2011).

${ }^{11} \mathrm{~T}$. Pierre, Physics of Plasmas 23, 042110 (2016), http://dx.doi.org/10.1063/1.4947564.

${ }^{12} \mathrm{~S}$. Jaeger, Étude théorique et expérimentale des instabilités basses fréquences dans un plasma en champs magnétique et éléctrique croisés, Ph.D. thesis, Université de Provence (2010).

${ }^{13}$ A. Simon, Physics of Fluids 6, 382 (1963).

${ }^{14}$ F. C. Hoh, Physics of Fluids 6, 1184 (1963)

${ }^{15}$ B. M. Annaratone, A. Escarguel, T. Lefevre, C. Rebont,

N. Claire, and F. Doveil, Physics of Plasmas 18, 032108 (2011).

${ }^{16}$ R. Decoste, Review of Scientific Instruments 56, 806 (1985).

${ }^{17}$ R. Granetz and P. Smeulders, Nuclear Fusion 28 (1988).

${ }^{18}$ L. C. Ingesson, P. J. Böcker, R. Reichle, M. Romanelli, and P. Smeulders, J. Opt. Soc. Am. A 16, 17 (1999).

${ }^{19}$ M. Anton, H. Weisen, M. Dutch, W. Linden, F. Buhlmann, R. Chavan, B. Marletaz, P. Marmillod, and P. Paris, Plasma Physics and Controlled Fusion 38, 1849 (1996).

${ }^{20}$ R. W. P. Mc Whirter, "Plasma diagnostic techniques," (New York Academic Press, 1965) p. 206.

${ }^{21}$ M. J. Druyvesteyn, Zeitschrift für Physik 64, 781 (1930).

${ }^{22}$ R. C. Woods and I. D. Sudit, Physical Revue E 50, 2222 (1994). 